\title{
The Educational Value Study on Regular Script of Chinese Characters
}

\author{
Tian-chen SONG ${ }^{1}$ and Fan-lei MENG ${ }^{2, a,{ }^{*}}$ \\ ${ }^{1,2}$ Linyi University, Feixian, Shandong, China \\ alydxmfl@126.com
}

Keywords: Regular script, Educational value, Position, Education path.

\begin{abstract}
Calligraphy has always been an important element of traditional Chinese culture, and the main practitioners of calligraphy culture inheritance are young students. When we appeal to educators and culture leaders to think about the way of educational demonstration on specialization of calligraphy shills, clear position is needed. The society, the news media, educational institutions, calligraphy community organizations, and all levels of calligraphy associations are obliged to provide direction guidance for the educational function of calligraphy, which means the calligraphy should be based on the regular script form, while the emersion of variety forms of calligraphy are also advocated. Here we will study the education path of regular script from aspect of teaching and social practice.
\end{abstract}

\section{Introduction}

Regular script, also known as standard characters, the evolution of it can be split into two forms: the Northern dynasty inscriptions and the Tang dynasty inscriptions. In broader terms, the regular script also includes seal character and official character, both of which are serious, elegant in letterforms. Now, for calligraphy beginners, no matter young or old, regular script is the foundation, is a must. All calligraphy beginners should start from regular script; only with a well-built foundation can the calligraphy learners become someone one day.

\section{The Historical Status of Regular Script}

The regular script holds a specific position in the long history of Chinese calligraphy art. It initiated in the end of Han Dynasty, popular during the Wei, Jin and The Southern and Northern Dynasties, and came to its culmination in Tang Dynasty. Regular script approximately initiated in the late Han Dynasty and the early Wei Dynasty (the early $3^{\text {rd }}$ century [1]. According to the literature No.1\&2 unearthed in Mawangdui Tomb, the "embryo of regular script" in the silk manuscript had already begun to take shape. The early regular script is similar to the official script to a rather large extent, so it is also called regular-style official script. The famous calligrapher, Zhongyao(Wei Dynasty), is known as the founder of regular script. His fonts are a little tabular, his strokes are massive and plain, all these make his works special and distinctive. By the Jin Dynasty, people follow the steps of Han Dynasty and Wei Dynasty; calligraphy talents emerged in succession, regular script becoming more and more mature and perfect. Wang Xizhi and Wang Xianzhi are great calligraphers in Eastern Jin Dynasty, they develop the ancient calligraphy techniques, make bold innovations, and make the regular script what they are today, and create rule and model for later generations [2]. The masterpieces of Wang Xizhi in regular script includes Huang Ting Jing( Scripture of 
the yellow court ), Yueyi Dissertation, Inscriptions of Dutiful Daughter Cao'E, while Wang Xianzhi has 13 Lines of the Godness of the Luo. The famous Northern dynasty inscriptions include Twenty Inscriptions in Longmen, Epitaph of Zhangxuan, Inscriptions of Zhang Menglong and so on. By the Sui Dynasty, the northern and southern China reunited under the Emperor Wen(Yang Jian), meanwhile, after 300 years of evolution in Wei, Jin , the South and North Dynasty, regular script has been already ripe gradually, and the trend of integration between the south and north is growing, it may be said that the integration of the north and the south in calligraphy has influenced the calligraphy art in Tang Dynasty. Longcang Temple Inscriptions, Qifa Temple Inscriptions, Inscriptions of Beauty Dong, The Epitaph of Su Xiaoci and so on, are all famous inscriptions in Sui Dynasty. These inscriptions on tablet are fine in strokes, elegant and finely developed in type-font, and are rather close to the regular script in Tang Dynasty[3].Tang Dynasty is the period when regular script reached maturity and perfection. To some extent, there are many calligraphers good at regular script after the period of Tang Dynasty, while none of them has ever been able to do better than people of Tang Dynasty. The history of regular script of Tang Dynasty can be split into three periods: in early Tang Dynasty, people follow the path of Wang Xizhi and Wang Xianzhi, and advocates the beauty of thin and hard strokes; famous calligraphers during this period are represented by $\mathrm{Yu}$ Shinan, $\mathrm{Ou}$ Yangxun, Chu Suiliang. In Resurgence Period of Tang Dynasty, Yan Zhenqing is the best, the most innovative calligraphers in Tang Dynasty. Calligraphers in this period advocate innovation, and fat and strong strokes, which is a result of the atmosphere of that time, as can be seen from the statue of Buddha and people's aesthetics in body, plumpness is the standard of beauty. The third period, is middle Tang Dynasty, lasts from Emperor Tai to Emperor Wen. During this period, the representative in calligraphy is Liu Gongquan. He absorbs the advantage of both Yan Zhenqing and Ou Yangxun, and makes his own innovations. He is famous for thin yet forceful strokes.

In the whole, regular script in Tang Dynasty have different basic features, rounded or square shape, thin or fat strokes. However, in the structure of regular script, it can be roughly split into only two forms, tight structures with tilted horizontal strokes and broad structures with horizontal strokes. Besides, the calligraphic style in this period is same, beauty of grace and harmony can be seen in every finely developed font, this, also shows the perfection and maturity of regular script in Tang Dynasty.

\section{The Educational Value Proposition of Regular Script}

Calligraphy has always been an important element in traditional Chinese culture. The practitioners of cultural heritage are mainly the young students. However, now, even though calligraphy amateurs and calligraphy associations of various levels in society have good intentions and great enthusiasm, while their works or exhibitions are caviar to the general public, they cannot understand the works. In this case, it's much harder to arouse the passion and enthusiasm of the young students for calligraphy.

Wang Qishan once said bluntly in the fifth plenary session of the $18^{\text {th }}$ Central Commission for Discipline Inspection: some leading cadres cannot write regular script well, they just skip to the running script, cursive, some even should frame their own works, and send to others. Certainly, these words direct against those unprofessional people who not only love showing, but also are corrupt. However, this also reflects social reality that the contemporary calligraphic attitude is not rigorous. Under the influence of rapid economic growth, and the impact of multi-culture, the social 
atmosphere is rather impetuous. As for writing, some adult learners cannot calm themselves down, start from the regular script, one stroke by one stroke.

Especially in today's world, the commercialization of art indeed makes someone impetuous, they push for quick profit, put cart before horse, flatter themselves with only few hours of learning, dare to express their opinions without studying the principle of calligraphy, see themselves as innovator without knowing the rule, publicize their personality without mastering the essence of calligraphy. So, the way to learn calligraphy is to choose a correct start, with peaceful mind, a correct start will help to make qualitative change, while peaceful mind will help to inspire you, eliminate all distractions, to achieve qualitative change and get inspiration in this subtle universe.

Clear proposition is needed when we appeal to educators and cultural leaders to think about the ways in educational demonstration from the angle of specialization of calligraphic techniques, so the society, medium, educational institutions, calligraphic community and calligraphy associations of all levels should provide direction guidance on the educational function of calligraphy, which means the artistic emersion should be based on the regular script form, while variety forms of calligraphy are also advocated.

\section{The Educational Value of Calligraphy}

It is generally acknowledged among calligraphy learners that the regular script is the base, the only way, but many people will avoid it, why? Because lots of time and energy are needed to learn regular script well, while people are chasing effective and fast pace of life, pushing for profits under multicultural background, as a result, the utilitarian predicament "skip to running script and cursive script" occurs.

The reason why beginners should spend lots of time on regular script has matters to do with "technique". Proficient techniques and profound foundation are the base of calligraphy, the absence of either will make it nearly impossible for calligraphy learners to enter into higher level. Both of them are needed to exert the artistic conception, spirit and interests into utmost. As ancient people said:" the gentleman will pay attention to the major part while the villain gaze at the minor part. Calligraphy beginners must keep their feet down to earth, step by step, stay calm; only in this way can they enter into higher level. The regular script is the most ideal style of characters to enhance ability in composure [4].

Regular script has high utility. Because it is rigorous, solemn, easy to read and understand, the inscriptions on the stone tablet to say praises in temple or palace are required to be written in regular script, to show solemnity.

Regular script is the basic teaching skill an elementary school teacher should have. As a teacher, especially an elementary school teacher, regular script is a required writing font. Particularly for Chinese teacher, they shoulder the task of teaching students to read and write new words. According to cognition rule and characteristics of elementary students, teachers must use and write standard Chinese characters.

Regular script will help to cultivate temperament. In modern society, the pace of life is fast, the interpersonal relationships are complicated, after work, if you can sit down and write several regular scripts carefully, you may feel peaceful, your nerve system will be relaxed, emotions will be balanced; you will feel happy when you writes well, and then, all the troubles will be nothing for you. The writing is indeed a joy of life.

Writing regular script helps to exercise strokes. For calligraphy art itself, the "strength" is the common beauty, any kind of writing font or style must be backed with strong stroke. The strike is a comprehensive artistic effect, it is not only about how you 
grab the writing brush, use your wrist. It also about the thickness, depth and shape of stroke, the square or rounded angle, the middle or the side angle when one use the writing brush, the rhythm and writing trend of the writing brush, it's also about structure of character. All above elements can be exercised in writing regular script.

Writing regular script can help to cultivate skills in steady, delicate and perfect stipple. Writing regular script requires every stipple to be perfect and have three-dimensional sense. If we observe the regular script strokes of ancient people carefully, we will find that they are trying to seek "vertical strokes" from out-of-vertical strokes, straight lines from out-of-straight lines, no matter what kind of shape they are, the strokes themselves are full of postures of human, while all these subtle changes can be fully shown in regular script.

Writing regular script can help to cultivate prudent ability. The fantasy of regular script lies in that it pursues thrill and inconceivability from straight lines, the relative balance of gravity from unequalled posture.

\section{The Way to Realize Educational Function of Regular Script}

Vigorously promote the practice of basic skills of regular script in normal colleges and universities. In the base of educators training-normal colleges, teachers should advance their basic calligraphy skills carefully, and students should practice their basic skills of regular script hard. History is the guide, the young students must learn from experiences of great calligraphers such as Zhong Yao and Wang Xizhi, to abandon the blundering state of mind, then build solid base of regular script. Take the community as carrier, guide the development of regular script through varieties of competitions. Found college students' calligraphy associations, organize specialized class in calligraphy, set up elective courses on regular script; provide opportunity and space for regular script based displaying during varieties of calligraphy competitions.

Enhance professional skills of part-time calligraphy teachers in primary schools Strengthen regular training of part-time calligraphy teachers in primary school, and advocate the spirit of putting regular script at first, vigorous promote writing standard Chinese character, hold all kinds of regular script competitions among primary amd middle schools, set up calligraphy exhibition hall, organize students to observe and study, and display new works regularly, so as to create good environment for calligraphy learning.

Appeal to social cultural leaders for regular script — oriented mechanism. Among varieties of competitions hold by different medium or calligraphy associations, the regular script based reward system must be built. Calligraphy learners should be advocated to begin with regular script, and get to know the way to use writing brush and ink in the process of practicing regular script, get to know the rhythm and writing trend in writing, get to feel, to know the rounded or square shape, the stroke, the wrist force, master different handwriting skills, so as to get different calligraphic emersions.

\section{Summary}

Chairman $\mathrm{Xi}$ pointed out, that the excellent traditional Chinese culture is the most powerful soft power, also is the fertile soil that socialism with Chinese characteristics deeply rooted in. Chinese calligraphy is an essential component of excellent traditional culture, regular script is the base of calligraphy, the source of calligraphy, now, to define the role of educational value of regular script should be put at priority. The appeals for 
the inheritance of regular script will help to rectify the impetuous social atmosphere generated from rapid economic growth under the multicultural background.

\section{Reference}

[1] Meng Baoyue, Reason Analysis of Tidiness of Tang Dynasty Regular Script [D] Nanjing Normal University, 2006.

[2] Zhang Shaohua, On Calligraphy [J] Journal of Jianghan University (Social Science Edition), 1985 (1).

[3] Liu Tianqi, Research on Inscriptions of Gravestone's Cover in Sui \& Tang Dynasties [D] Xi' an Academy of Fine Arts, 2009.

[4] Li Hanning, Analysis on Calligraphic Education Countermeasures in Higher Normal Universities [J] Art Education, 2005(6). 